\title{
Motion capture as an instrument in multimodal collaborative learning analytics
}

\author{
Milica Vujovic ${ }^{[0000-0003-0963-7182]}$, Simone Tassani ${ }^{[0000-0002-8652-2314]}$, \\ Davinia Hernández-Leo [0000-0003-0548-7455] \\ Universitat Pompeu Fabra, Barcelona \\ milica.vujoviceupf . edu
}

\begin{abstract}
In this paper, we describe an exploratory study where we investigate the possibilities of motion capture system as an instrument to consider in multimodal analyses of face-to-face collaborative learning scenarios. The goal is to understand to what extent motion capture can facilitate certain measurements leading to collaborative learning indicators that are currently time-consuming to achieve with other instruments. We focus on the simultaneous measurement of known physical collaboration indicators such as gaze direction, the distance between learners and the speed of movement/reactions. The study considers a lab setting simulating a classroom scenario based on the Jigsaw collaborative learning flow pattern, which proposes a sequence of activities with changes in group size and formation. Preliminary results indicate a high degree of applicability of the system in measuring these indicators, with certain limitations for gaze direction measurements. With appropriate marker position on the participants, the system is able to automatically provide desired measurements with satisfactory precision. Additionally, with a small number of additional markers, we were able to determine the way students used working surfaces (shared desks).
\end{abstract}

Keywords: Motion Capture System, Multimodal Learning Analytics, CSCL.

\section{Introduction}

Despite there is accumulated evidence about the benefits of collaborative learning, there are still many research questions about what happens in the collaboration process and what makes it more effective. In face-to-face settings, there is emerging research that uses multimodal learning analytics (MMLA) to identify indicators of fruitful collaboration. Some indicators have been already identified, such as collaborative will [1], equality and mutuality [2], symmetry [3], synchrony of groups' actions and gaze [4], the reaction time of participants to the actions of members of the group [5] or the distance between learners (DBL) [6] etc. Multimodal measures leading to these indicators are diverse (video, audio, physiological data, ...) and generate large amount of data, which require significant time-consuming analysis. In this paper, we select concrete physical collaboration indicators such as gaze direction (GD), the distance between learners (DBL) and the movement speed/reaction (MS), and propose and evaluate the 
application of a motion capture system (MCS) with the objective to simultaneously measure these indicators and accelerate the analysis process (Fig. 1).

a)

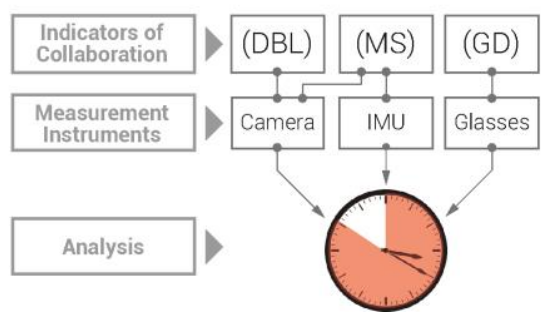

b)

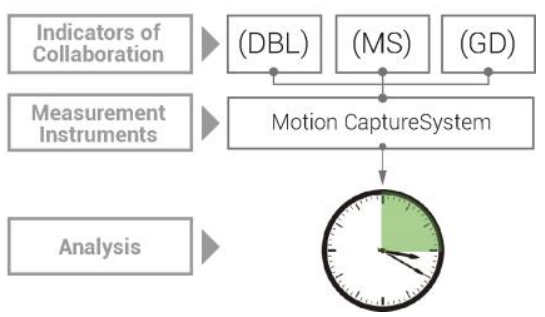

Fig. 1. Substituting various instruments with Motion Capture to accelerate the analysis.

\section{Motion Capture in Collaborative Learning Analytics}

To illustrate and evaluate the possibilities of MCS for collaborative learning analytics, we use a scenario based on the Jigsaw pattern [7] where students are grouped into small independent groups and where each student is assigned with a specific role. Students are then regrouped on the basis of their roles in order to gain expertise and share that expertise with other members of the group. Such a context is a complex environment where there are constant interactions of participants and group size and formation transformations. The monitoring of participant behaviour and factors that influence the collaboration process is a demanding task. As aforementioned, by selecting three indicators (DBL, MS, GD) we propose to substitute different sensors, like cameras, Inertial Measurement Units, eye-tracking glasses etc. with MCS. In comparison to other technologies that address the issue of movement detection (such as possibility of detecting pose using web camera, or deep learning algorithms for depth perception), we found that they face problems such as tracking bigger group of people or having nor so high accuracy rate. Regarding ethical issues, we have informed participants on details of the experiment and collected a consent form.

Application of motion capture systems is wide and cross-disciplinary [8]. The system applied in this study uses reflective markers and infrared cameras, where markers are placed on objects whose movement we want to detect. Because of the reflective surface, the cameras recognize them as points in space, based on which we get the desired physical parameters. The main advantage of the system is that it is possible to develop a marker protocol fully adapted to the needs of the research.

\section{Evaluation of the Motion Capture System}

We studied to what extend MCS represents a useful MMLA tool in the analysis of collaborative learning indicators in a Motion Caption Laboratory (left-down, Fig. 2), where we run an experimental protocol for a pair of three member groups that participated in one Jigsaw session. Movement analysis was performed using eight cameras 
BTS Smart-DX 700, 1.5 MP 125 fps (BTS S.p.A., Milan, Italy). A custom marker protocol was developed to follow the movement of the subjects analyzed using headbands with 5 non-aligned markers for each of the participants. Two lateral markers were placed at the level of the ears, the other two were at the back of the head at different levels and one marker on the top of the head (Head Motion Marker Protocol). Middle points between the rear and lateral markers were identified, together with the vector passing through these points. A calibration process was performed to identify the GD.

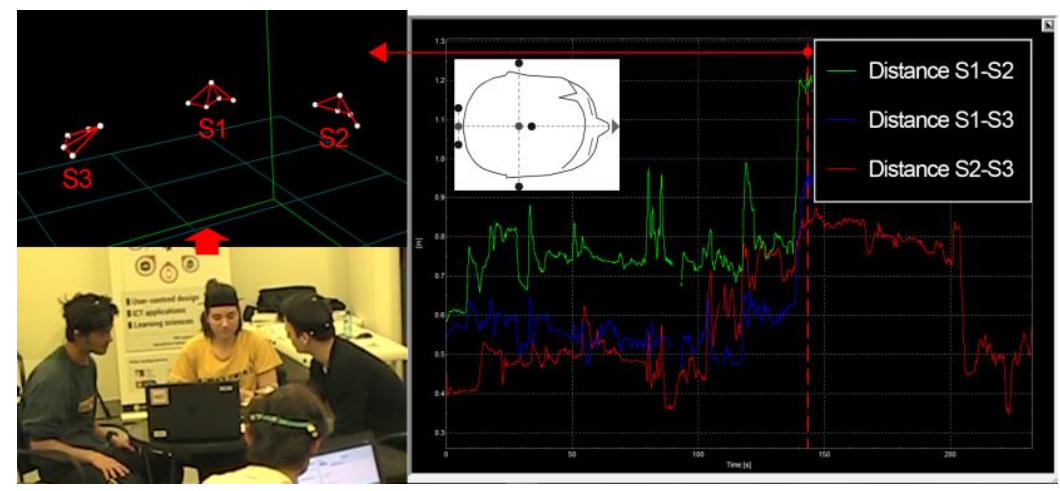

Fig. 2. Reconstruction of markers and presentation of the DBL in specific point in time

Nine measurements of five minutes each were performed to cover the three phases of the collaborative Jigsaw activity. The analysis tool enabled us to calculate the DBL and the MS within a few minutes based on the marker positioned at the top of the head and by selecting two operators (distance and derivatives). The GD calculation required additional operators, which took more time.

The Figure 2 shows the reconstruction of the markers (left-top, Fig. 2), capture from a video recording (left-down, Fig. 2), position of markers (middle, Fig. 2), and a graph that displays the DBL (left-down, Fig. 2) during one recording (300 seconds). One of the moments during the activity was randomly selected (red dashed line) to show that the tool can display the values at any given moment and various indicators at the same time.

The scope of this study is efficiency and comprehensiveness, which we analyzed through the speed of analysis and obtaining the desired indicators. The most time consuming phase is the reconstruction of markers. Calculation of results in the case of two indicators (DBL and MS) takes several minutes, while the calculation of the GD takes 20-30 minutes. Video recordings are included and used to control the obtained results.

\section{Discussion and Conclusion}

The use of MCS as an instrument for multimodal analysis of collaborative learning has proved to be effective in the context of this study. The results of the study indicate the advantage of ease of detection of DBL and MS, due to the use of only one marker and the rapid analysis of data. With these indicators, the number of participants in the study 
does not affect the quality of the recording and analysis. Identifying the GD is done based on the position of the head and ignoring the movement of the eyes, which represents a limitation that is difficult to overcome without the use of additional resources. With all indicators, a comprehensive display of data is possible and clearly visible, which is an additional quality of the system. The constraints that occur, in addition to the precise detection of GD, are the connection of the system to the physical environment, which possible to overcome with different interventions, such as displacing system outside the laboratory or using mobile motion capture systems. All these interventions have disadvantages in terms of time or price, but they can be justified by beneficial contributions in the field of multimodal analysis. Future work should focus on additional features useful to analyze collaboration processes (sitting arrangement, use of the desk surface) and that can be easily labeled, recorded and analyzed.

\section{Acknowledgements}

This project has received funding from the European Union's Horizon 2020 research and innovation programme under the Marie Skłodowska-Curie grant agreement No. 713673. Milica Vujovic has received financial support through the "la Caixa" INPhINIT Fellowship Grant for Doctoral studies at Spanish Research Centres of Excellence, "la Caixa" Banking Foundation, Barcelona, Spain. This work has been partially supported by the National Research Agency of the Spanish Ministry of Science, Innovations and Universities MDM-2015-0502, TIN201453199-C3-3-R, TIN2017-85179-C3-3-R.

\section{References}

1. Pijeira-Díaz, H. J., Drachsler, H., Järvelä, S., Kirschner, P. A.: Investigating collaborative learning success with physiological coupling indices based on electrodermal activity. ACM Proceedings of the Sixth International Conference on Learning Analytics \& Knowledge, 6473 (2016).

2. Damon, W., Phelps, E.: Critical distinctions among three approaches to peer education. International Journal of Educational Research, 13.1. 9-19 (1989).

3. Dillenbourg, P.: Collaborative learning. Pergamon. Amsterdam. (1999).

4. Schneider, B., Blikstein, P.: Unraveling students' interaction around a tangible interface using multimodal learning analytics. Journal of Educational Data Mining, 7.3 89-116 (2015).

5. Raca, M., Tormey, R., Dillenbourg, P.: Sleepers' lag-study on motion and attention. ACM Proceedings of the Fourth International Conference on Learning Analytics and Knowledge, 36-43 (2014).

6. Spikol, D., Ruffaldi, E., Cukurova, M.: Using multimodal learning analytics to identify aspects of collaboration in project-based learning. 12th International Conference on Computer Supported Collaborative Learning (CSCL), 263-270 (2017).

7. Aronson, E., Patnoe, S.: Cooperation in the Classroom: The Jigsaw Method. 3rd ed. Pinter \& Martin, Ltd., London (2011).

8. Park, S. W., Park, H. S., Kim, J. H., Adeli, H.: 3D displacement measurement model for health monitoring of structures using a motion capture system. Measurement 59, 352-362 (2015). 